

\title{
L'hypothèse du champ moléculaire et la propriété ferromagnétique
}

Pierre Weiss

\section{To cite this version:}

Pierre Weiss. L'hypothèse du champ moléculaire et la propriété ferromagnétique. J. Phys. Theor. Appl., 1907, 6 (1), pp.661-690. 10.1051/jphystap:019070060066100 . jpa-00241247

\section{HAL Id: jpa-00241247 https://hal.science/jpa-00241247}

Submitted on 1 Jan 1907

HAL is a multi-disciplinary open access archive for the deposit and dissemination of scientific research documents, whether they are published or not. The documents may come from teaching and research institutions in France or abroad, or from public or private research centers.
L'archive ouverte pluridisciplinaire HAL, est destinée au dépôt et à la diffusion de documents scientifiques de niveau recherche, publiés ou non, émanant des établissements d'enseignement et de recherche français ou étrangers, des laboratoires publics ou privés. 


\section{L'HYPOTHĖSE DU CHAMP MOLÉGULAIRE ET LA PROPRIÉTÉ FERROMAGNÉTIQUE;}

Par M. Pierre WeisS $\left({ }^{1}\right)$.

M. Langevin (2) a donné récemment une théorie qui rend compte d'une manière remarquable des propriétés des corps diamagnétiques et paramagnétiques.

Il suppose que chaque molécule d'un gaz paramagnétique a un moment magnétique différent de zéro, résultant de l'addition géométrique des moments magnétiques des trajectoires fermées que les corpuscules décrivent avec de grandes vitesses autour du reste de l'atome. Un champ extérieur agissant sur ces molécules tend à les orienter, l'agitation thermique et les chocs qui l'accompagnent tendent à rétablir constamment l'état de désordre primitif. Il en résulte un équilibre statique pour lequel le moment magnétique par unité de volume, c'est-à-dire l'intensité d'aimantation I, est fonction du quotient du champ $\mathrm{H}$ par la force vive d'agitation thermique, qui est proportionnelle à la température absolue T. M. Langevin trouve en effet :

$$
\frac{\mathrm{I}}{\mathrm{I}_{0}}=\frac{\operatorname{ch} a}{\operatorname{sha}}-\frac{1}{a}
$$

où la signification de a est donnée par :

$$
a=\frac{\mu \cdot \mathrm{H}}{r \mathrm{~T}}
$$

$I_{0}$ représente la somme de tous les moments magnétiques des molécules dans 1 centimètre cube, c'est-à-dire l'intensité d'aimantation

- que l'on obtiendrait si tous ces moments étaient dirigés parallèlement; $\mu$, le moment magnétique d'une molécule, et $r \mathrm{~T}$, deux fois la force vive correspondant à un degré de liberté de la molécule.

La relation (1) est représentée par la courbe de la fig. 1. Elle met en évidence un accroissement de l'aimantation d'abord proportionnel à $a$, c'est-à-dire à $\mathrm{H}$, pour une température donnée, et dans les champs très intenses une aimantation à saturation $\mathrm{I}_{0}$ correspondant

(1) Conférence faite à la Société française de Physique, le 4 avril 1907.

(2) P. Langevin, Ann. Chim. Phys., so série. t. V. p. 70 et suiv.; 1903.

J. de Phys., $4^{\circ}$ série, t. VI. (Septembre 1907.) 
à l'alignement complet des aimants élémentaires. M. Langevin montre que les champs qui seraient nécessaires pour constater les écarts de la proportionnalité sont hors de proportion avec ceux que nous savons produire. Et l'expérience donne, en effet, un coefficient d'aimantation spécifique \% (susceptibilité rapportée à l'unité de masse) constant pour les corps paramagnétiques.

Ce coefficient, la théorie le donne inversement proportionnel à la température absolue, d'accord avec Pierre Curie qui a découvert cette propriété sur l'oxygène et montré qu'elle s'étend à un certain nombre d'autres corps. Si j'insiste sur ce point, c'est que, dans la suite, nous considérerons ce mode de dépendance de la température comme caractéristique de l'état paramagnétique.

Curie, dans son travail sur les Propriétés magnétiques des corps $\dot{a}$ diverses températures, a été vivement frappé de la très grande ressemblance des courbes représentant l'intensité d'aimantation en fonction du champ et de la température avec celles qui représentent la densité d'un fluide en fonction de la pression et de la température. Il résulte de leur comparaison que l'état paramagnétique est analogue à l'état gazeux, l'état ferromagnétique à l'état liquide. M. Langevin, indiquant la voie à suivre pour étendre aux corps ferromagnétiques la théorie qu'il a donnée des corps paramagnétiques, conclut de même : "On voit donc quelle est l'importance des actions mutuelles entre les molécules qui rendent seules possible la saturation magnétique encore extrêmement éloignée, pour le même champ extérieur, dans le cas des substances faiblement magnétiques. "

Champ moléculaire. - Je me propose de montrer ici que l'on peut fonder une théorie du ferromagnétisme sur une hypothèse extrêmement simple concernant ces actions mutuelles. Je suppose que chaque molécule éprouve de la part de l'ensemble des molécules environnantes une action égale à celle d'un champ uniforme NI proportionnel à l'intensité d'aimantation et de même direction qu'elle. On pourrait donner à NI le nom de champ intérieur pour marquer l'analogie avec la pression intérieure de van der Waals. Ce champ, en effet, s'ajoutant au champ extérieur, va rendre compte de la grande intensité d'aimantation des corps ferromagnétiques au moyen de lois des corps paramagnétiques, comme la pression intérieure, s'ajoutant à la pression extérieure, rend compte de la forte densité des liquides en invoquant la compressibilité des gaz. Mais cette expression donnerait lieu à de fréquentes confusions. Je lui ai préféré celle de champ 
moléculaire. On sera conduit à considérer, ici comme ailleurs, une sphère d'activité moléculaire.

J'admets, en outre, qu'il n'y a pas d'autres actions qui dérivent d'une énergie potentielle de rotation des molécules, c'est-à-dire qui se traduisent par des moments exercés sur celles-ci. En d'autres termes, à part les actions mutuelles exprimée̊s par le terme NI, les rotations sont, dans le corps ferromagnétique, aussi libres que dans un gaz. L'énergie potentielle de translation n'est l'objet d'aucune hypothèse déterminée.



FIG. 1.

Supposons, d'abord, que le champ intérieur existe seul et montrons que, de même que les liquides peuvent exister sous une pression extérieure nulle (et même négative), les corps ferromagnétiques peuvent prendre une intensité d'aimantation finie en l'absence de champ extérieur. L'équation (2) devient :

$$
\mathrm{I}=a \frac{r \mathrm{~T}}{\mu \mathrm{N}} .
$$

Cette équation est représentée dans la fig. 1 par la droite OA. L'intensité d'aimantation devant satisfaire à (1) et à (3), les points d'intersection de la courbe et de la droite donneront les valeurs de 1 . Une solution est évidente : c'est $\mathrm{J}=\mathrm{o}, a=0$, et par suite $\mathrm{H}=\mathrm{o}$. Mais il est facile de voir que ce n'est pas celle-ci, mais celle qui est donnée par le point A qui correspond à un état stable. Imaginons, en effet, que, modifiant l'orientation des molécules par une intervention directe, on donne à l'intensité d'aimantation une valeur un peu inférieure à $\mathrm{AA}^{\prime}$. La valeur de $a$, donnée par la droite, sera alors un peu supérieure à celle que donne la courbe pour la même intensité 
d'aimantation. Or ces valeurs de $a$ sont proportionnelles au champ moléculaire et au champ exigé par la formule de Langevin. Le premier l'emporte donc et l'aimantation remontera jusqu'à la valeur $\mathrm{AA}^{\prime}$. De même, une aimantation un peu supérieure à $\mathrm{AA}^{\prime}$ décroîtra jusqu'à cette valeur.

Nous aurons à mettre d'accord cette conception assez imprévue d'une aimantation finie obtenue dans un champ nul avec les faits expérimentaux du ferromagnétisme dans lesquels le champ extérieur joue un rôle considérable, et pour cela nous invoquerons plus loin les propriétés ferromagnétiques des cristaux.

Pour le moment, remarquons seulement que, par suite de la petitesse de la susceptibilité paramagnétique, il faudrait des champs énormes pour augmenter encore cette aimantation spontanée, elle ne peut donc être égale qu'à ce que l'on appelle communément l'intensité d'aimantation à saturation à la température considérée $\mathrm{T}$.

Variation thermique du ferromagnélisme. - Quand la température s'élève, le coefficient angulaire de la droite OA augmente proportionnellement à $\mathrm{T}$ et, par conséquent, l'intensité d'aimantation diminue. Elle s'annule pour la température $\theta$ à laquelle la droite est tangente à la courbe au point $O$. $\theta$ est la température absolue de perte du ferromagnétisme spontané. Les deux équations (1) et (3) peuvent être considérées comme représentant la loi de variation de l'intensité d'aimantation en fonction de la température au moyen de la variable auxiliaire $a$.

En remarquant que, à la limite $a=0$, on a $: \frac{\mathrm{I}}{\mathrm{I}_{0}} \cdot \frac{1}{a}=\frac{1}{3}$, il vient :

$$
\theta=\frac{\mu \mathrm{NI}_{0}}{3 r} \text {. }
$$

L'équation (3) peut donc être mise sous la forme:

$$
\frac{\mathrm{T}}{0}=\frac{3}{a} \frac{\mathrm{I}}{\mathrm{I}_{0}}
$$

La loi de variation de l'intensité d'aimantation avec la température est donc la même pour tous les corps, exprimée par les états correspondants caractérisés par les valeurs de $\frac{\mathrm{T}}{\theta}$ et $\frac{\mathrm{I}}{\mathrm{l}_{0}}$. Elle est représentée par la courbe de la fig. 2; sa parenté est évidente avec les courbes expérimentales connues jusqu'à présent, mais elle s'en distingue par une variation plus rapide de l'aimantation aux basses 
températures. La raison en est qu'avec des champs assez faibles, dans lesquels on a opéré jusqu'ici, la saturation est atteinte dans le voisinage de 0 seulement, tandis qu'aux températures plus basses l'aimantation est restée en deçà de la saturation. Pierre Curie, dans un champ de 1300 gauss, a obtenu pour le fer à la température ordinaire une intensité d'aimantation égale à 1700 . Plus tard, du Bois et Taylor Jones (') ont montré que cette valeur n'est pas encore la saturation et que l'intensité d'aimantation du fer continue à croìtre entre 10000 et 13000 gauss pour atteindre, dans ce dernier champ, la valeur $18 \check{0} 0$.



Fig. 2.

Vérification expérimentale. - Je me suis adressé pour la vérification de cette loi à la magnétite, dont une étude antérieure m'avait appris qu'elle est sensiblement saturée dans un champ de 500 gauss, et j’ai opéré dans un champ de 8300 gauss.

La méthode de mesure que j'ai emplơée est nouvelle. Elle consiste à tailler la substance sous forme d'un ellipsoïde et à la suspendre au moyen d'un ressort de torsion dans le champ horizontal d'un électroaimant mobile autour d'un axe vertical. On mesure ainsi le couple exercé par l'aimant sur la substance. Soient $\mathrm{N}_{1}$ et $\mathrm{N}_{2}$ les coefficients

(') Du Bois et TaYlor Jones, Elektrot. Zeilschr., t. XYII. p. J44́ ; 1896. 
démagnétisants principaux de l'ellipsoïde pour les axes horizontaux $\mathrm{O} x$ et $\mathrm{O} y$, et $\mathrm{V}$ le volume, le couple est :

$$
\mathrm{C}=\mathrm{V} \text {. I . H } \sin (\alpha-\varphi),
$$

où $\alpha$ est l'angle du champ de l'électro-aimant, $\varphi_{i}$ celui de l'intensité d'aimantation avec $\mathrm{O} x$. Mais le champ résultant du champ extérieur et du champ démagnétisant de l'ellipsoïde a la même direction que l'intensité d'aimantation, donc:

$$
\frac{\mathrm{H} \cos \alpha-\mathrm{N}_{1} \mathrm{I} \cos \varphi}{\cos \varphi}=\frac{\mathrm{H} \sin \alpha-\mathrm{N}_{2} \mathrm{I} \sin \varphi}{\sin \varphi},
$$

d'où :

$$
\mathrm{C}=\mathrm{V}\left(\mathrm{N}_{2}-\mathrm{N}_{1}\right) \mathrm{I}^{2} \sin \varphi \cos \varphi .
$$

Ce couple a un maximum qui correspond à $\varphi=40^{\circ}$ :

$$
\mathrm{C}=\frac{1}{2} \mathrm{~V}\left(\mathrm{~N}_{2}-\mathrm{N}_{1}\right) \mathrm{I}^{2} \text {. }
$$

Il est égal, à une constante près, au carré de l'intensité d'aimantation. Il suffit donc de faire tourner l'aimant jusqu'à ce que la déviation soit maximum, et de mesurer ce maximum. Il n'est nécessaire de connaître ni la valeur exacte, ni l'orientation du champ. J'ai pris soin de tailler l'ellipsoïde de magnétite de façon qu'au moment de la mesure l'intensité d'aimantation ait la direction d'un axe binaire du cristal, c'est-à-dire d'un maximum relatif d'aimantation. Il suffit que cette condition soit réalisée avec une approximation grossière pour que les couples d'origine magnétocristalline aient une influence tout à fait subordonnée.

Cette mesure a été faite à des températures variant de celle de la neige carbonique $\left(-79^{\circ}\right)$ à celle de la disparition du ferromagnétisme $\left(+587^{\circ}\right)$. Les points ont été marqués dans la même figure que la courbe théorique ( $f g .2)$ dans laquelle l'échelle des abscisses a été déterminée au moyen de l'ensemble des quatre observations correspondant aux températures les plus élevées, de façon à faire coïncider les températures de disparition du ferromagnétisme théorique et expérimentale. L'échelle des ordonnées a été choisie de manière à placer sur la courbe théorique le deuxième point, correspondant à la température ordinaire. On voit que le premier point seul s'écarte un peu de la courbe. La vérification de la théorie est donc tout à fait satisfaisante. 
Explication des phénomènes magnétocristallins par l'hypothèse du champ moléculaire. - Les propriétés magnétiques du cristal de pyrrhotine, que j'ai étudié expérimentalement dans un travail antérieur $\left({ }^{1}\right)$, peuvent se résumer de la manière suivante :

Le cristal possède une direction privilégiée $\mathrm{O} x$ dans laquelle l'intensité d'aimantation a une grandeur constante, égale à l'intensité d'aimantation à saturation, quelle que soit la grandeur du champ. Si l'on fait agir dans la même direction un champ croissant de sens contraire à l'intensité d'aimantation, celle-ci change brusquement de sens au moment où le champ dépasse une certaine valeur $\mathrm{H}_{c}$ qui porte le nom de champ coercitif. Le cycle d'aimantation correspondant à cette direction est donc un rectangle. L'intensité d'aimantation n'est susceptible de prendre que deux valeurs, celles de la saturation positive et négative. Si parfois elle paraît prendre des valeurs intermédiaires, c'est parce qu'une certaine fraction de la matière est aimantée dans le sens positif et le reste dans le sens négatif et que l'on observe la différence.

Les phénomènes qui se produisent dans cette direction $\mathrm{O} x$, que j'ai appelée direction de facile aimantation, sont donc irréversibles.

Quand on fait agir le champ dans une direction oblique sur $\mathrm{O} x$, l'aimantation s'écarte de cette direction en conservant une grandeur constante et en restant dans le plan de base du prisme orthorhombique de la pyrrhotine, auquel j'ai, pour cette raison, donné le nom de plan magnétique. Cette déviation de l'aimantation est réversible. Tout se passe comme si, dans la direction $\mathrm{O} y$ perpendiculaire à $\mathrm{O} x$ et contenue dans le plan magnétique, s'exerçait un champ démagnétisant $n \times \mathrm{I} y$, provenant de la structure cristalline et proportionnel à la composante de l'intensité d'aimantation suivant $\mathrm{O} y$. Pour faire coïncider l'intensité d'aimantation avec l'axe $O y$, il faut que le champ extérieur, dirigé lui aussi suivant $\mathrm{O} y$, soit au moins égal à la valeur maxima de ce champ démagnétisant, qui a été trouvée égale à : $n I=7300$ gauss. Des expériences encore inédites ont montré que ce champ se retrouvait avec la mème valeur dans un grand nombre de fragments provenant de plusieurs échantillons ; c'est donc une constante caractéristique.

La propriété du plan magnétique n'est pas rigoureuse.Quand on donne au champ une composante importante dans la direction $\mathrm{O} z$

(1) J. de Phys., $4^{\text {e }}$ série, t. IV, p. 469 et 829 ; 1903. 
perpendiculaire à ce plan, on obtient une faible composante de l'intensité d'aimantation parallèle à $\mathrm{O} z$ et proportionnelle à cette composante du champ. J'en avais conclu que la pyrrhotine, ferromagnétique pans le plan magnétique, est paramagnétique perpendiculairement à ce plan. Je préfère ici un énoncé différent pour les mêmes faits. Tout se passe dans le plan $x \mathrm{O} z$ comme dans le plan $x \mathrm{O} y$, avec la différence que le champ démagnétisant structurai est beaucoup plus grand dans la direction $\mathrm{O} z$ que dans la direction $\mathrm{O} y$, sa valeur maxima étant de l'ordre de grandeur de $n^{\prime} \mathrm{l}=150000$ gauss.

Au lieu de supposer, comme je l'ai fait implicitement, que le champ moléculaire est le même dans toutes les directions, j'admettrai maintenant que l'édifice cristallin possède trois plans de symétrie rectangulaires coïncidant avec les plans de coordonnées, et que chacune des composantes du champ moléculaire est proportionnelle à la composante correspondante de l'intensité d'aimantation avec un coefficient différent, $\mathrm{N}_{1}>\mathrm{N}_{2}>\mathrm{N}_{3}$, pour chacun des trois axes.

Alors le champ moléculaire n'a plus en général la direction de l'intensité d'aimantation, sauf lorsque celle-ci est dirigée suivant l'un des axes. Elle ne pourra donc se diriger d'elle-même que suivant $\mathrm{O} x, \mathrm{O} y$ ou $\mathrm{O} z$, et cette orientation ne correspondra à un équilibre stable que pour l'axe $(\mathrm{O} x)$ pour lequel le coefficient $\left(\mathrm{N}_{1}\right)$ est le plus grand, comme nous allons nous en rendre compte.

Quand un champ extérieur s'ajoute au champ moléculaire, l'aimantation prend une direction coïncidant avec le champ résultant, ce qui s'exprime par :

$$
\frac{\mathrm{H}_{x}+\mathrm{N}_{1} \mathrm{I}_{x}}{\mathrm{I}_{x}}=\frac{\mathrm{H}_{y}+\mathrm{N}_{2} \mathrm{I}_{y}}{\mathrm{I}_{y}}=\frac{\mathrm{H}_{z}+\mathrm{N}_{3} \mathrm{I}_{z}}{\mathrm{I}_{z}} .
$$

Supposons I et $\mathrm{H}$ contenus dans le plan des XY, et appelons $\alpha$ et $p$ les angles du champ et de l'intensité d'aimantation avec OX, alors :

$$
\mathrm{HI} \sin (\alpha-\varphi)-\left(\mathrm{N}_{1}-\mathrm{N}_{2}\right) I^{2} \sin \varphi \cos \varphi=0 .
$$

Cette équation est identique à celle par laquelle j'ai résumé antérieurement les propriétés expérimentales de la pyrrhotine dans le plan des XOY, si l'on pose $\left(\mathrm{N}_{1}-\mathrm{N}_{2}\right) \mathrm{I}=n \mathrm{I}=7300$ gauss. L'hy pothèse que nous venons de faire rend donc compte des propriétés expérimentales de la pyrrhotine dans le plan XOY. Nous avons déjà dit que ces propriétés ne diffèrent de celles du plan XOZ que par la 
grandeur de la constante. On aura pour ce dernier:

$$
\left(\mathrm{N}_{1}-\mathrm{N}_{\ddot{3}}\right) \mathrm{I}=n^{\prime} \mathrm{I}=150000 \text { gauss. }
$$

L'équation (7) exprime que le couple exercé par le champ extérieur sur l'intensité d'aimantation H.I $\sin (\alpha-\vartheta)$, qui tend à éloigner celle-ci de l'axe des $x$, est égal au couple dù à la structure $\left(\mathbf{N}_{1}-\mathbf{N}_{2}\right) I^{2} \sin \varphi \cos \varphi$, qui l’y ramènerait si l'on supprimait le champ extérieur. La position d'équilibre correspondant à l'orientation de l'aimantation suivant $\mathrm{O} x$ n'est donc stable que si $\mathrm{N}_{1}$ est le plus grand des trois coefficients.

L'étude des propriétés magnétocristallines ne donne donc que les différences entre les champs moléculaires principaux. On verra plus loin que la considération simultanée des propriétés magnétiques en deçà et au delà de la température 0 de perte du ferromagnétisme spontané donne la valeur du champ moléculaire lui-même. Puisque l'aimantation se dirige d'elle-même suivant l'axe où il est maximum, ce sera le champ moléculaire maximum, $\mathrm{N}_{1} \mathrm{I}$, que l'on aura ainsi déterminé.

C'est à l'expérience de montrer si le schéma du cristal magnétique caractérisé par les quatre nombres $\mathrm{I}, \mathrm{H}_{c}, n \mathrm{I}, n^{\prime} \mathrm{I}$ est applicable à d'autres corps. Le peu que l'on sait de la magnétite cristallisée n'y contredit pas, à condition d'admettre que les cristaux étudiés jusqu'à présent étaient formés par la juxtaposition ou la pénétration de cristaux plus simples de diverses orientations. L'un des champs démagnétisants principaux $n$ I semble être, pour cette matière, égal à 500 gauss environ.

On dispose d'un plus grand nombre de données sur le fer, qui cristallise, comme la magnétite, en cristaux d'apparence cubique. Mais les expériences ont été faites sur les fers industriels, qui consistent en des enchevêtrements de cristaux sur lesquels on ne peut déterminer que des valeurs moyennes. Encore ces moyennes n'ont-elles une signification simple que quand aucune direction n'est favorisée, ce qui n'a pas lieu en général dans les métaux laminés ou passés à la filière. Mais il se peut fort bien qu'une circonstance particulière au fer, le passage à l'état $\gamma$ à $920^{\circ}$, intervienne ici d'une manière avantageuse, grâce à la recristallisation qui l'accompagne, comme l'a montré $\mathrm{M}$. Osmond. Il suffirait donc de recuire à une température suffisamment élevée pour rétablir l'isotropie apparente. 
Synthèse des propriétés du fer, champs faibles, cycles d'hystérèse. - Quoi qu'il en soit, je me propose de montrer que l'on peut, en attribuant au cristal élémentaire de fer des propriétés analogues à celles du cristal de pyrrhotine et en construisant de toutes pièces les courbes d'aimantation d'un milieu formé de cristaux orientés en tous sens, retrouver les propriétés expérimentales et donner la raison de quelques-unes de leurs particularités jusqu'à présent inexpliquées.

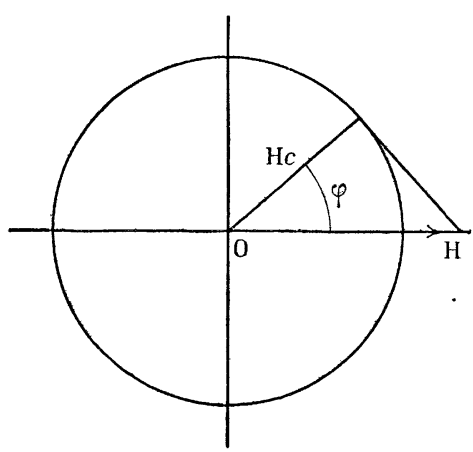

FIG. 3.

Supposons d'abord que, restant dans les champs faibles, on ne considère que le phénomène irréversible et que l'on néglige la déviation réversible de l'aimantation à partir de la direction de facile aimantation dans chaque cristal. Quand la matière est à l'état neutre, l'ensemble des vecteurs aimantation des différents cristaux élémentaires sera réparti. sur une sphère avec une densité constante. Lorsque le champ $\mathrm{H}$, agissant suivant une direction fixe $\mathrm{OH}$, dépasse le champ coercitif $\mathrm{H}_{c}$, toutes les intensités d'aimantation contenues à l'intérieur d'un cône ayant $\mathrm{OH}$ pour axe et un demi-angle au som-

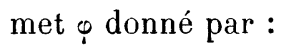

$$
\cos \varphi=\frac{\mathrm{H}_{c}}{\mathrm{H}},
$$

qui étaient primitivement dirigées du côté négatif, changeront de signe, et l'intensité d'aimantation résultante sera:

$$
\jmath=\frac{\mathrm{I}}{2} \sin ^{2} \varphi=\frac{\mathrm{I}}{2}\left[1-\left(\frac{\mathrm{H}_{c}}{\mathrm{H}}\right)^{2}\right],
$$

où I représente l'intensité d'aimantation quel'on obtiendrait si toutes les directions de facile aimantation coïncidaient avec $\mathrm{O} x$. 
Si l'on arrête la croissance du champ à la valeur $\mathrm{H}$ et qu'on le fasse ensuite osciller entre $+\mathrm{H}$ et $-\mathrm{H}$, l'intensité d'aimantation reprendra périodiquement la valeur maxima I, donnée par cette équation. Celle-ci représente donc le lieu des sommets des cycles. C'est une cubique dont la partie utile ressemble grossièrement à une branche d'hyperbole équilatère. Elle admet l'asymptote horizontale $I=\frac{I}{2}$, pour laquelle la distance entre la courbe et l'asymptote est infiniment petite du second ordre, et l'asymptote verticale $\mathrm{H}=0$. Elle rencontre l'axe des abscisses au point $\mathbf{H}=\mathrm{H}_{c}$.

Supposons maintenant, au lieu de partir de l'état neutre, que tous les points figuratifs aient été rassemblés par l'effet d'un champ très fort, du côté négatif de la sphère. Commençons ensuite la description d'un cycle en faisant varier le champ de $-\infty$ à des valeurs positives croissantes. Il ne se passera rien, et l'aimantation restera $-\frac{1}{2}$ tant que le champ sera inférieur à $+H_{c}$. Puis l'intensité d'aimantation décrira une courbe partant du point $\left(-\frac{\mathrm{I}}{2},+\mathrm{H}_{c}\right)$, identiqué à la courbe des sommets des cycles que nous avons déjà trouvée et possédant les mêmes asymptotes, mais dont les ordonnées sont portées à une échelle double. Ce sera, avec la portion de la droite $-\frac{I}{2}$ déjà décrite, la moitié du cycle limite, correspondant aux variations du champ de $-\infty$ à $+\infty$. Il se complète par symétrie par rapport à l'origine.

On voit facilement que les cycles correspondant à des variations du champ d'amplitude finie, de $-\mathrm{H} \grave{a}+\mathrm{H}$, s'obtiennent en utilisant la courbe des sommets des cycles déjà trouvée et en faisant passer par ces sommets des droites horizontales et la courbe du cycle limite déplacée verticalement.

Pour pousser plus loin l'approximation, il faut tenir compte de la déviation que le champ fait éprouver à l'intensité d'aimantation à partir de sa direction initiale. Connaissant les deux champs démagnétisants principaux $H_{D}^{\prime}=\left(N_{1}-N_{2}\right)$ I et $H_{D}=\left(N_{1}-N_{3}\right)$ I, et la répartition des points figuratilis sur la sphère, on possède tous les éléments du calcul.

Soient $\alpha_{1}, \alpha_{2}, \alpha_{3}$ les cosinus directeurs du champ extérieur $H$ par rapport aux axes rectangulaires d'un cristal élémentaire, $\varphi_{1}, \varphi_{2}, \varphi_{3}$ 
ceux de lintensité d'aimantation. La composante de l'aimantation dans la direction du champ est donnée par:

$$
y=\mathrm{I}\left(\alpha_{1} \varphi_{1}+\alpha_{2} \varphi_{2}+\alpha_{3} \varphi_{3}\right)
$$

avec

$$
\frac{\mathrm{H} \alpha_{1}+\mathrm{N}_{1} \mathrm{I} \varphi_{1}}{\varphi_{1}}=\frac{\mathrm{H} \alpha_{2}+\mathrm{N}_{2} \mathrm{I} \varphi_{2}}{\varphi_{2}}=\frac{\mathrm{H} \alpha_{3}+\mathrm{N}_{3} \mathrm{I} \varphi_{3}}{\varphi_{3}},
$$

équation qui exprime que l'intensité d'aimantation et le champ résultant du champ extérieur et du champ moléculaire ont la même direction.

Éliminant $\varphi_{1}, \varphi_{2}, \varphi_{3}$, on a la loi de l'aimantation dans la direction du champ pour ce cristal élémentaire. Le développement de çette loi en fonction des puissances de $\mathrm{H}$ est:

$$
\begin{aligned}
y=\mathrm{I}\left[\alpha_{1}+\mathrm{H}\left(\frac{\alpha_{2}^{2}}{\mathrm{H}_{\mathrm{D}}}+\frac{\alpha_{3}^{2}}{H_{\mathrm{D}}^{\prime}}\right)-\frac{\mathrm{H}^{2}}{1.2}\left(\frac{3 \alpha_{1} \alpha_{3}^{2}}{\mathrm{H}_{\mathrm{D}}^{\prime 2}}+\frac{3 \alpha_{1} \alpha_{2}^{2}}{\mathrm{H}_{\mathrm{D}}^{2}}\right)\right. \\
\left.+\frac{\mathrm{H}^{3}}{1.2 .3}\left(\frac{12 \alpha_{1}^{2} \alpha_{3}^{2}}{\mathrm{H}_{\mathrm{D}}^{\prime 3}}+\frac{12 \alpha_{1}^{2} \alpha_{2}^{2}}{\mathrm{H}_{\mathrm{D}}^{3}}\right)-\ldots\right] .
\end{aligned}
$$

Lorsque les directions de facile aimantation sont figurées par des points couvrant l'hémisphère positif avec une densité constante, une double intégration donne la loi de l'aimantation d'une substance isotrope en apparence :

$$
\text { (11) } \begin{aligned}
J & =\mathrm{I}\left[\frac{1}{2}+\frac{\mathrm{H}}{3}\left(\frac{1}{\mathrm{H}_{\mathrm{D}}}+\frac{1}{\mathrm{H}_{\mathrm{D}}^{\prime}}\right)-\frac{3}{16} \mathrm{H}^{2}\left(\frac{1}{\mathrm{H}_{\mathrm{D}}^{2}}+\frac{1}{\mathrm{H}_{\mathrm{D}}^{\prime 2}}\right)\right. \\
& \left.+\frac{2}{15} \mathrm{H}^{3}\left(\frac{1}{\mathrm{H}_{\mathrm{D}}^{3}}+\frac{1}{\mathrm{H}_{\mathrm{D}}^{\prime 3}}\right)-\frac{1}{384} \mathrm{H}^{\prime}\left(\frac{1}{\mathrm{H}_{\mathrm{D}}^{\prime}}+\frac{10}{\mathrm{H}_{\mathrm{D}}^{2} \mathrm{H}_{\mathrm{D}}^{\prime 2}}+\frac{1}{\mathrm{H}_{\mathrm{D}}^{\prime 4}}\right)+\ldots\right] .
\end{aligned}
$$

Dans le cas, au contraire, où la matière partirait d'un état initial neutre, les puissances impaires de $H$ subsisteraient seules, avec les mêmes coefficients.

Le coefficient de la première puissance de H représente la susceptibilité initiale étudiée par lord Rayleigh (1) et dont il a montré qu'elle se conserve dans toutes les petites variations réversibles de l'aimantation produites par les variations du champ de petite amplitude, même lorsque l'aimantation a atteint une fraction notable de l'intensité de saturation. La théorie rend compte, par conséquent, de la constance de cette susceptibilité réversible. Mais elle ajoute une conséquence aisément vérifiable par l'expérience. Cette susceptibi-

(1) Lord Rayleigh, Philosophical Magazine, XXIII, p. 225-245; 1887. 
lité initiale peut s'écrire :

$$
\mathrm{K}_{0}=\frac{1}{3}\left(\frac{1}{\mathrm{~N}_{1}-\mathrm{N}_{2}}+\frac{1}{\mathrm{~N}_{1}-\mathrm{N}_{3}}\right)
$$

elle est donc indépendante de la température, si les coefficients $N_{1}$, $\mathrm{N}_{2}, \mathrm{~N}_{3}$ sont eux-mêmes indépendants de la température comme nous l'avons supposé. Les expériences sur la variation de la susceptibilité avec la température (') dans les champs faibles semblent pouvoir s'accorder avec cette conséquence de la théorie. En général, elles donnent un accroissement relativement faible de la susceptibilité quand la température s'élève. Ainsi Ewing trouve que, pour un fil de fer écroui, l'intensité varie de $\mathbf{2 , 1 4}$ à 2,23 dans le champ terrestre quand la température varie de $6^{\circ}$ à $100^{\circ}$. La difficulté d'interprétation de cette expérience et des autres analogues vient de ce que la région de la susceptibilité initiale constante devient rapidement très restrcinte aux températures élevées, comme cela est visible sur certaines courbes d'Hopkinson $\left({ }^{2}\right)$. Il serait nécessaire de faire de nouvelles expériences en explorant exactement, aux températures élevées, l'étendue de la région de la susceptibilité initiale.

La fig. 4 représente les cycles tracés par le procédé ci-dessus indiqué avec les constantes $\mathrm{I}_{\mathrm{M}}=1900, \mathrm{H}_{c}=1,2$, et corrigés au moyen de la susceptibilité rézersible et du terme quadratique du développement $\left.(11){ }^{3}\right)$, en admettant $H_{D}=120$ gauss, $H_{D}^{\prime}=\infty$. (Nous verrons en effet plus loin que l'un des champs $\mathrm{H}_{\mathrm{D}}, \mathrm{H}_{\mathrm{D}}^{\prime}$ doit être très grand, sa valeur numérique n̈importe pas ici.) Ces constantes ont été choisies de manière à reproduire aussi exactement que possible les courbes expérimentales $(f g .5)$ qui ont été calquées sur une planche du mémoire classique d'Ewing ('). Il n'est pas nécessaire d'insister sur la ressemblance des lignes ascendantes, plus particulièrement frappante pour le cycle limite, ni sur l'inclinaison constante des lignes descendantes dans le voisinage immédiat des summets des cycles, qui est l'expression de la constance de la suscep-

(1) Ewrag, Nagnetic Incluction in Iron, $3^{\mathrm{e}}$ éd.; London, 1900, p. 166.

(2) Ewra, loc. cit., p. 17', fig. 82 .

(3) Le phénomène reversible modifie extrêmement peu le phénomène irréversible auquel il sajoute, tant que les champs restent faibles; voir J. de Phys., $4^{\circ}$ série, t. IV, p. 829 et suiv.

(4) Ewing, Phil. Trans., t. CLxx VI, p. ว̈23:18s6; - Magnetic Incluction, $3^{\circledR}$ éd., p. 106 , fig. 50 . 
674

WEISS

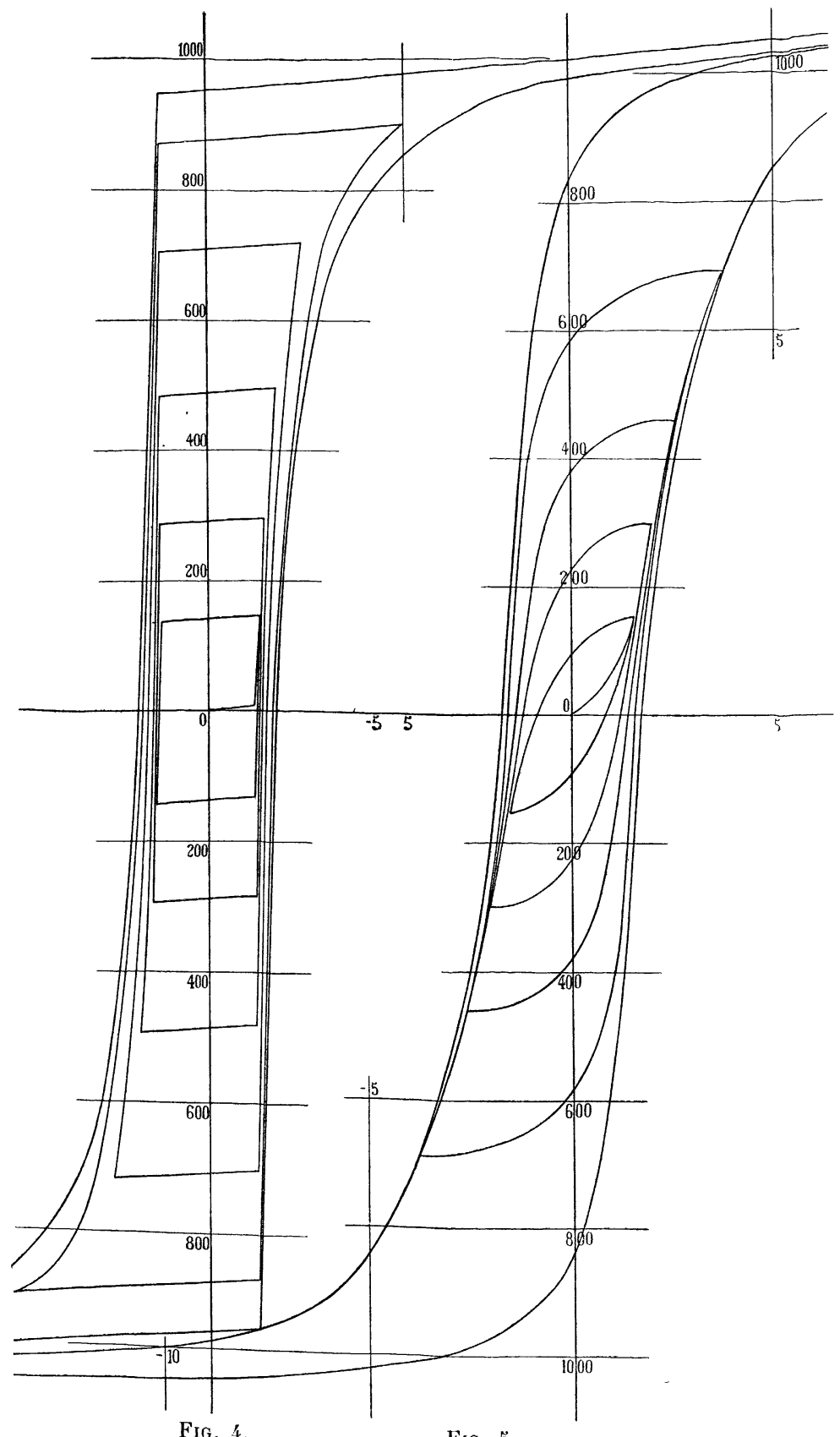

FIG. 4.

FIG. วั. 
tibilicé réversible de lord Rayleigh; mais il y a lieu de noter quelques différences entre la théorie et l'expérience et d'en donner l'explication.

$1^{\circ}$ Les sommets des cycles de moyenne grandeur sont plus voisins de l'ascendante du cycle limite dans la fig. $ّ$ que dans la fig. 4. Ce caractère des courbes expérimentales est souvent plus accentué encore que dans l'exemple que nous avons choisi. Il arrive fréquemment, en effet, que les sommets des petits cycles sont coupés par les ascendantes des cycles un peu plus grands, comme dans la fig. 6. La raison semble en être la suivante: tandis que la totalité de la matière intervient dans la description du cycle limite, plus le cycle est petit, moins grande est la fraction de la matière qui est intéressée par le phénomène irréversible, et tout se passe cornme si cette fraction était diluée dans une matière plus faiblement magnétique. Il en résulte une influence démagnétisante par suite de laquelle les petits cycles sont relativement plus inclinés que les grands.

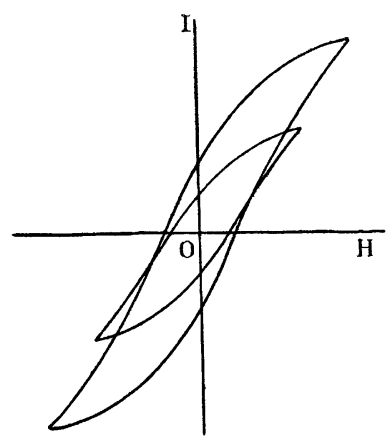

Fig. 6.

$2^{\circ}$ Il y a une différence considérable dans l'aspect des petits cycles que la théorie donne strictement réversibles, et d'aire nulle, tant que $\mathrm{H}$ oscille entre des limites comprises entre $\mathrm{H}_{c}$, et ensuite, pour $\mathrm{H}$ un peu plus grand, de forme presque rectangulaire, tandis que l'expérience fournit les courbes arrondies dont lord Rayleigh(1) a montré que, dans un intervalle assez étendu, elles peuvent être assimilées à des paraboles. Pour expliquer cette apparition des phénomènes irréversibles dès les champs les plus faibles, on peut admettre qu'à côté des molécules en plus grand nombre dont la sphère d'activité est com-

(1) Lord Rayleigh, loc. cit. 
plète, il y en a d'autres pour lesquelles la sphère d'activité est entamée par la surface du corps ou par les surfaces de discontinuité intérieures, d'autant plus importantes que le grain de la structure cristalline est plus fin et que la matière est moins pure. Or, pour qu'un groupe d'aimants moléculaires, solidaires par leurs actions mutuelles, ait le champ coercitif normal $\mathrm{H}_{c}$ que nous avons seul considéré jusqu'à présent, il est probable qu'il faut que la sphère d'activité soit intacte pour un grand nombre d'entre eux. Dans le cas contraire, le pivotement irréversible à $180^{\circ}$ se produira pour un champ $\mathrm{H}_{c}^{\prime}<\mathrm{H}_{c}$.

C'est dans ces portions de matière irrégulières que nous chercherons l'origine du terme en $\mathrm{H}^{2}$ des paraboles de lord Rayleigh, que la théorie, qui ne conduit qu'à des termes impairs pour l'aimantation dans les champs $<\mathrm{H}_{c}$, ne donnait pas. L'importance relative très variable du terme en $\mathrm{H}$ et du terme en $\mathrm{H}^{2}$, donnée par l'expérience, vient à l'appui de cette interprétation, mettant en évidence le caractère accidentel de ce dernier. En effet, lord Rayleigh a trouvé pour un fil de fer de Suède écroui :

$$
\mathrm{I}=6,4 \mathrm{H}+5,1 \mathrm{H}^{2} .
$$

En faisant des expériences analogues sur un autre échantillon de fil de fer de Suède écroui, j'ai trouvé(') :

$$
\mathrm{I}=9,46 \mathrm{H}+1,44 \mathrm{H}^{2} \text {. }
$$

D'autres auteurs donnent des valeurs encore plus divergentes pour le coefficient de $\mathrm{H}^{2}\left({ }^{2}\right)$.

L'existence de ces matières accessoires à champ coercitif moindre explique aussi, au moins en partie, les arrondis qui remplacent l'angle vif de la descendante des grands cycles.

$3^{\circ}$ Quand le champ passe d'une valeur quelconque à une valeur moindre, sans changement de signe, la théorie indique que la variation concomitante de l'aimantation doit ètre réversible. L'expérience n'est d'accord avec cette conséquence que pour une décroissance infiniment petite du champ. Quand cette variation est finie, quand elle consiste, par exemple, dans un retour du champ à zéro, on observe les phénomènes bien connus représentés par la fig. 7 , où l'existence

(1) Eclairage électrique. VIII, p. $436 ; 1896$.

(2) Holbors, Winkelmann Handbuch, 2e éd., V1, p. 236. 
de la boucle $\mathrm{AB}$ témoigne de l'irréversibilité. Or, pour qu'il se produise un phénomène irréversible, il est nécessaire qu'en certains points au moins de la matière le champ ait le même signe que la variation de l'aimantation. lci, où l'aimantation décroît, le champ doit donc être négatif. Cela ne peut avoir lieu que si les actions mutuelles entre les cristaux élémentaires, dans lesquels l'aimantation est diversement orientée, sont assez fortes pour renverser le champ en certains points.

Ce phénomène doit être plus important pour certaines structures cristallines que pour d'autres. Il sera, toutes choses égales d'ailleurs, plus fréquent pour les substances à grande intensité d'aimantation. Ewing ( $\left.{ }^{1}\right)$ signale un échantillon de nickel de champ coercitif relativement considérable pour lequel une branche de courbe correspondant à $\mathrm{AB}$ est très approximativement réversible.



FIG. 7.

On voit donc que la théorie rend compte de la forme des cycles autant qu'il semble possible de le faire et que les divergences qui restent s'expliquent d'une manière très plausible. Notons encore quelques renseignements quantitatifs qui se déduisent de la théorie.

L'ordonnée à l'origine du cycle limite est égale à la moitié de l'intensité d'aimantation à saturation. Or la plus grande intensité d'aimantation qui ait été mesurée, égale à 1800 , est probablement encore un peu inférieure à celle de la saturation. Je relève, dans le mémoire

(1) Ewing, Phil. Trans., t. CLXXIX., p. 327; 1889.

J. de Phys., $4^{\mathrm{e}}$ série, t. Vl. (Septembre 1907.) 
d'Ewing, l'ordonnée à l'origine de plusieurs cycles limites de fer doux :

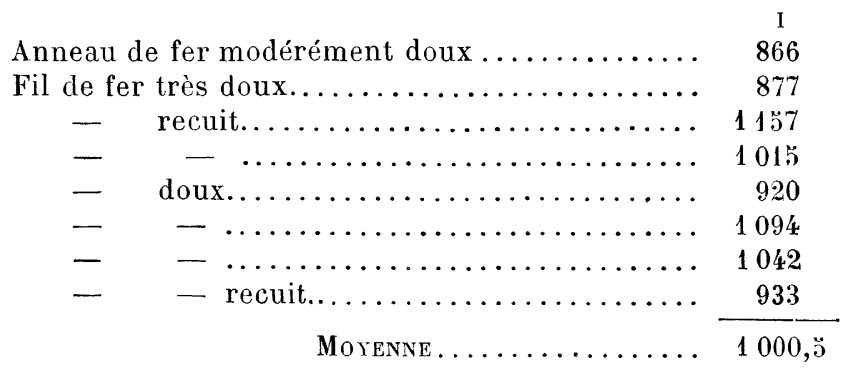

Ceflte concordance est assez satisfaisante, eu égard à ce que l'on ne sait jusqu'à quel point les fers satisfont à la condition d'isotropie apparente.

L'abscisse du point d'intersection du cycle limite avec l'axe des $\mathrm{H}$ est $\mathbf{H}_{c} \sqrt{2}$. C'est ce que l'on appelle communément le champ coercitif. On doit, par contre, obtenir $\mathrm{H}_{c}$ par l'intersection du lieu des sommets des cycles avec cet axe. Pour tenter cette vérification, il faudrait disposer d'une substance n'ayant pas d'éléments de champ coercitif moindre. Ou bien, approximativement, on peut extrapoler la partie sensiblement rectiligne du lieu des sommets des cycles jusqu'à l'axe des champs. Les expériences que l'on possède jusqu'à présent vérifient grossièrement cette propriété.

Champs moyens et champs intenses. - Quand on examine la portion de la courbe d'aimantation d'un fer très doux qui est comprise entre $H= \pm 20$ gauss, par exemple, on y remarque, après l'accroissement rapide de l'aimantation opérée dans la région du cycle d'hysstérèse, une augmentation plus lente que l'on pourrait prendre pour un indice du voisinage de la saturation. Mais il n'en est rien, l'accroissement de l'aimantation est, dans cette région, du même ordre de grandeur que celui qui correspond à la susceptibilité initiale, et l'intensité d'aimantation est encore assez voisine de $\frac{\mathrm{I}}{2}$.

On a indiqué fréquemment comme intensité d'aimantation à saturation du fer une valeur voisine de 1 700. Mais, malgrél'intérêt considérable qui s'attache à la connaissance exacte de la loi d'approche de la saturation et qui a été signalé d'abord par du Bois, on ne possède encore que peu de données précises se rapportant à des champs supérieurs à 1000 gauss. Parmi les meilleures on peut citer 
celles de Roessler $\left({ }^{1}\right)$, dont la fig. 8 reproduit graphiquement une partie et met en évidence la physionomie caractéristique de la courbe d'aimantation dans les champs compris entre 600 et 1300 gauss. De ce qu'entre 800 et 1300 gauss l'aimantation ne croît que de $0,50 / 0$ de sa valeur, Roessler croit pouvoir conclure que la saturation est atteinte, mais la $f g .8$ montre que ses expériences s'accordent encore mieux avec l'hỵpothèse d'une croissance régulière de l'aimantation, à raison de 20 unités par 1000 gauss, dans les champs compris entre 600 et 1300 gauss.



Les expériences plus anciennes de du Bois $\left({ }^{2}\right)$ par la méthode magnétooptique, qui vont jusqu'à 1200 gauss, donnent de même un accroissement de l'aimantation de 25 unités environ par 1000 gauss aux environs de $\mathrm{H}=1000$.

L'aimantation continue en effet à croître, comme le montre le tableau suivant, emprunté à du Bois et Taylor Jones $\left({ }^{3}\right)$, qui contient les seules données numériques précises que nous ayons pour les champs très intenses.

(1) Roessler, Elektrotech. Zeitschr., t. XIV, p. 97, 114, 133, 149, 161; 1893.

(2) Du Bors, Phil. Mag., 5ै série, t. XXIX, p. $293 ; 1890$.

(3) Du Bois et TAylor Jones, Elektrot. Zeitschr., t. XVII, p. $543 ; 1896$. 


\begin{tabular}{rccc}
$H$ & \multicolumn{3}{c}{$J$} \\
\cline { 2 - 4 } 5000 & 1800 & 1625 & 1780 \\
10000 & 1830 & 1750 & 1820 \\
15000 & 1850 & 1770 & 1850
\end{tabular}

L'hypothèse que nous avons faite sur la constitution du fer permet de calculer, en s'appuyant sur les équations (8) et (9), la loi d'approche de la saturation. On trouve:

$$
\begin{aligned}
& \text { (13) I }-J=\frac{I^{3}}{15 \mathrm{H}^{2}}\left[\left(\mathrm{~N}_{1}-\mathrm{N}_{2}\right)^{2}+\left(\mathrm{N}_{1}-\mathrm{N}_{3}\right)^{2}-\left(\mathrm{N}_{1}-\mathrm{N}_{2}\right)\left(\mathrm{N}_{1}-\mathrm{N}_{\dot{3}}\right)\right] \\
& +\frac{\mathrm{I}^{4}}{3 \times 5 \times 7 \mathrm{H}^{3}}\left[\left(\mathrm{~N}_{1}-\mathrm{N}_{2}\right)\left(\mathrm{N}_{1}-\mathrm{N}_{3}\right)^{2}+\left(\mathrm{N}_{1}-\mathrm{N}_{2}\right)^{2}\left(\mathrm{~N}_{1}-\mathrm{N}_{3}\right)+\left(\mathrm{N}_{2}-\mathrm{N}_{3}\right)\left(\mathrm{N}_{2}-\mathrm{N}_{1}\right)^{2}\right. \\
& \left.\quad+\left(\mathrm{N}_{2}-\mathrm{N}_{3}\right)^{2}\left(\mathrm{~N}_{2}-\mathrm{N}_{1}\right)+\left(\mathrm{N}_{3}-\mathrm{N}_{1}\right)\left(\mathrm{N}_{3}-\mathrm{N}_{2}\right)^{2}+\left(\mathrm{N}_{3}-\mathrm{N}_{1}\right)^{2}\left(\mathrm{~N}_{3}-\mathrm{N}_{2}\right)\right]+\ldots
\end{aligned}
$$

La distance entre la courbe et l'asymptote est donc infiniment petite du second ordre. Le coefficient du premier terme peut s'écrire:

$$
\frac{\mathrm{I}}{1 \breve{J}}\left(\mathrm{H}_{\mathrm{D}}^{2}+\mathrm{H}_{\mathrm{D}}^{\prime 2}-\mathrm{H}_{\mathrm{D}} \cdot \mathrm{H}_{\mathrm{D}}^{\prime}\right) \text {. }
$$

La connaissance expérimentale de ce terme, jointe à celle de la susceptibilité initiale :

$$
\mathrm{K}_{0}=\frac{1}{3}\left(\frac{1}{\mathrm{H}_{\mathrm{D}}}+\frac{1}{\mathrm{H}_{\mathrm{D}}^{\prime}}\right)
$$

donne donc les deux champs démagnétisants principaux.

Les expériences dans les champs forts et dans les champs faibles n'ont jamais été faites sur la même matière. On ne saurait donc les associer pour une détermination numérique de $\mathrm{H}_{\mathrm{D}}$ et $\mathrm{H}_{\mathrm{D}}^{\prime}$. Mais on peut en déduire que ces champs démagnétisants sont très inégaux, le plus petit étant de l'ordre de grandeur de 20 à 100 gauss, le plus grand de plusieurs milliers de gauss. La susceptibilité initiale dépend alors presque exclusivement du premier, l'approche de l'asymptote du second.

En négligeant le premier, et en posant, par conséquent,

$$
\mathrm{N}_{1}=\mathrm{N}_{2}>\mathrm{N}_{3}, \quad\left(\mathrm{~N}_{1}-\mathrm{N}_{3}\right) \mathrm{I}=\mathrm{H}_{\mathrm{D}}^{\prime},
$$

la formule (13) devient :

$$
\mathrm{I}-\partial=\mathrm{I}\left[\frac{1}{15}\left(\frac{\mathrm{H}_{\mathrm{D}}^{\prime}}{\mathrm{H}}\right)^{2}-\frac{2}{105}\left(\frac{\mathrm{H}_{\mathrm{D}}^{\prime}}{\mathrm{H}}\right)^{3}+\ldots\right],
$$


et le calcul donne, dans ce cas particulier, une valeur nulle pour le terme suivant en $\left(\frac{\mathrm{H}_{\mathrm{D}}^{\prime}}{\mathrm{H}}\right)^{4}$, de sorte que les deux premiers termes peuvent représenter une portion de courbe relativement étendue.

Cette formule s'accorde avec l'allure générale des expériences de $\mathrm{du}$ Bois et Taylor Jones, et donne :

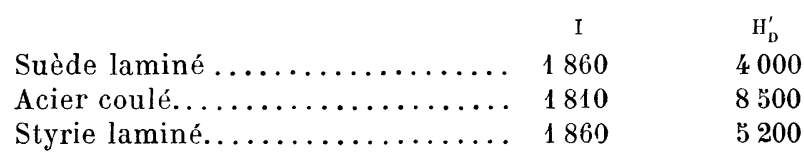

Mais il faudrait de nouvelles expériences, extrêmement précises, pour vérifier plus complètement cette théorie.

La grandeur du rapport $\frac{\mathrm{H}_{\mathrm{D}}^{\prime}}{\mathrm{H}_{\mathrm{D}}}$ montre que le fer, comme la pyrrhotine, possède un plan magnétique approché. On s'explique facilement, alors, la saturation apparente qui a été fréquemment observée dans le voisinage de 1000 gauss. Si le plan magnétique était rigoureux, la direction de l'aimantation tendrait, dans chaque cristal élémentaire, vers la projection du champ sur le plan magnétique. Sa valeur résultante, $\frac{\pi}{4} \mathrm{I}$, déterminerait la hauteur de l'asymptote de la courbe d'aimantation. Dans le cas où le plan magnétique est approché, il en résulte la faible inclinaison du palier rectiligne, que nous avons signalée d’après les expériences de Roessler ( $f g .8$ ) et de du Bois.

L'équation de la portion de courbe voisine de cette fausse asymptote peut être calculée. On trouve :

$$
\gamma=\frac{\pi}{4} \mathrm{I}+\frac{\mathrm{I}}{3} \underset{\mathrm{H}_{\mathrm{D}}^{\prime}}{\mathrm{H}}-\frac{\pi}{16} \mathrm{I} \underset{\mathrm{H}^{2}}{\mathrm{H}^{2}},
$$

et, en supprimant le dernier terme, on a la fausse asymptote elle-même. Cette droite peut être tracée assez facilement dans le voisinage de la courbe expérimentale, on en déduit une nouvelle détermination de 1 et de $\mathrm{H}_{\mathrm{D}}^{\prime}$. Les expériences de Roessler donnent ainsi :

$$
\mathrm{I}=\frac{4}{\pi} \cdot 166 \mathrm{~J}=2120
$$

et

$$
\mathrm{H}_{\mathrm{v}}^{\prime}=35000 \text {. }
$$

On trouve ainsi un champ démagnétisant principal notablement 
plus élevé que par le voisinage de la vraie saturation. Mais, eu égard à l'incertitude de l'isotropie apparente et de l'identité de la matière et à la petitesse des différences sur lesquelles repose cette détermination, il est déjà très satisfaisant de trouveru ne valeur du même ordre de grandeur. Une détermination analogue faite incidemment, dans un autre travail, sur un échantillon de fer doux, m'a donné :

$$
\mathrm{I}=1972, \quad \mathrm{H}_{\mathrm{D}}^{\prime}=7500
$$

valeurs qui concordent beaucoup mieux avec celles que nous avons déduites des expériences de du Bois et Taylor Jones.

Il est donc possible, en principe, de déterminer les constantes caractéristiques du cristal de fer $\mathrm{I}, \mathrm{H}_{c}, \mathrm{H}_{\mathrm{D}}, \mathrm{H}_{\mathrm{D}}^{\prime}$, par des expériences sur des échantillons isotropes en apparence, et de soumettre l'hypothèse sur la nature de ce cristal à un certain nombre de contrôles. 11 n’est pas douteux que des expériences dirigées spécialement vers ce but ne permettent des conclusions beaucoup plus nettes que celles que nous pouvons tirer ici.

Il serait sans doute préférable encore de suivre la voie inverse en tentant l'étude directe du fer cristallisé, malgré son extrême difficulté.

Les propriétés magnétiques du fer au delà de la température de perte du ferromagnétisme sponlané. - La théorie qui a été donnée ci-dessus pour la variation du ferromagnétisme en fonction de la température peut être étendue facilement au cas où le champ extérieur $\mathrm{H}_{e}$ est différent de zéro. L'équation (2) devient, en substituant:

$$
\mathrm{H}=\mathrm{H}_{\ell}+\mathrm{NI},
$$

et, en tenant compte de (1):

$$
\frac{\mathrm{T}}{\theta}=\frac{3}{a} \frac{\mathrm{I}}{\mathrm{I}_{0}}+\frac{3 \mathrm{H}_{e}}{a \mathrm{NI}_{0}} .
$$

Cette équation, combinée avec l'équation (1), donne, par l'intermédiaire de la variable auxiliaire $a$, la relation entre $\frac{\mathrm{T}}{\theta}$ et $\frac{\mathrm{I}}{\mathrm{I}_{0}}$ qui correspond au champ extérieur $\mathrm{H}_{e}$. Elle se distingue de la relation analogue pour $\mathrm{II}_{e}=$ o par l'adjonction à $\frac{\mathrm{T}}{\theta}$ d'un terme inversement proportionnel à $a$. Or, tant que $a$ est petit, cette quantité est égale à $3 \frac{\mathrm{I}}{\mathrm{I}_{0}}$, 
et par suite :

$$
\frac{\mathrm{T}}{\theta}-1=\frac{\mathrm{H}_{e}}{\mathrm{NI}}
$$

L'excès $\mathrm{T}-\Theta$ de la température sur la température de disparition du ferromagnétisme spontané est donc inversement proportionnel à l'intensité d'aimantation I qui persiste. La représentation graphique de la relation entre ces deux quantités est une hyperbole équilatère dont le paramètre est proportionnel au champ extérieur. La fig.9, empruntée au mémoire de Pierre Curie, montre que l'expérience confirme d'une manière remarquable cette théorie des phénomènes voisins de la température $\theta$.

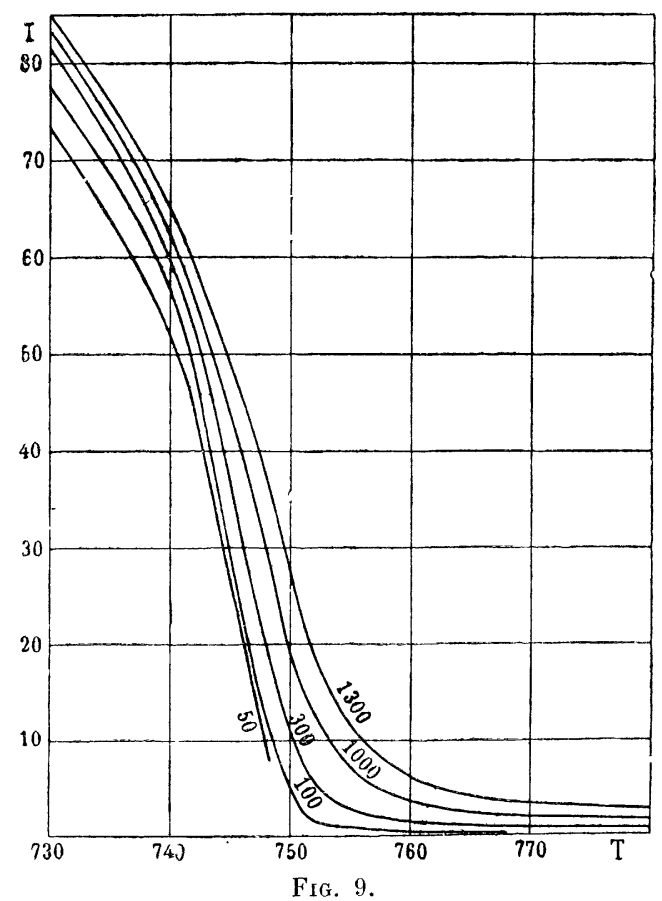

Il ne semble pas douteux que les autres substances ferromagnétiques ne se comportent comme le fer dans le voisinage de $\theta$. Curie l'a d'ailleurs établi pour plusieurs d'entre elles. En d'autres termes, elle passent de l'état de ferromagnétisme spontané (état $\alpha$ ) à celui du ferromagnétisme sollicité par le champ extérieur (état $\beta$ ), et il 
est possible que certaines substances, le nickel par exemple, n'existent qu'à ces deux états. Mais les expériences de Curie, qui se sont étendues à un intervalle de températures notable, lui ont fait retrouver par leurs caractères magnétiques deux autres états du fer dont le premier est le fer $\gamma$ de M. Osmond et le second, déjà aperçu par Ball, doit être appelé fer ò. Les expériences sont résumées schématiquement dans la $f g$. 10, dans laquelle les températures ont été portées en abscisses et les coefficients d'aimantation spécifique \% en ordonnées. Dans la région $\beta$, qui commence à $756^{\circ}$, le coefficient d'aimantation spécifique part de l'infini pour décroître suivant une loi exac-

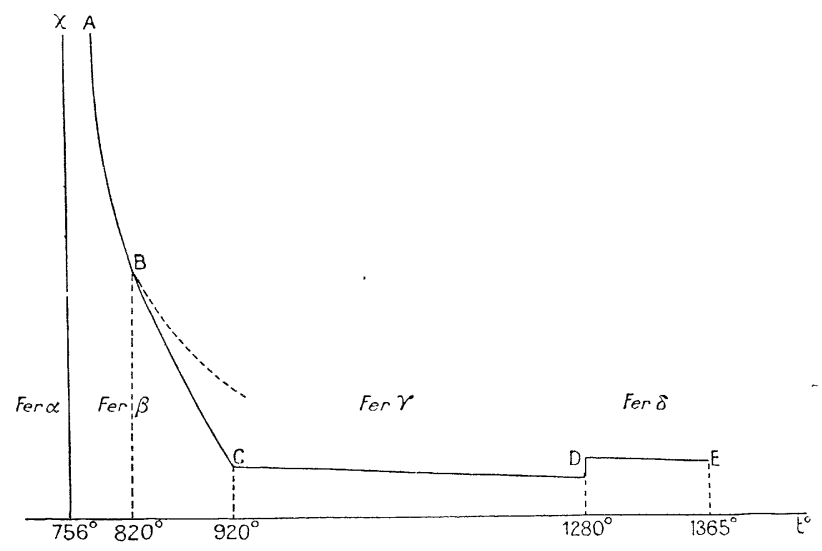

FIG. 10.

tement hyperboliquejusque vers $820^{\circ}$. Ensuite la décroissance est plus rapide. Il est probable qu'entre cette température et celle de $920^{\circ}$, à partir de laquelle commence l'état $\gamma$, a lieu une transformation progressive. A l'état $\gamma$, le fer possède une susceptibilité inversement proportionnelle à la température absolue, caractéristique de l'état paramagnétique. A $1280^{\circ}$, dernier changement d'état, le coefficient d'aimantation spécifique croît brusquement dans le rapport de 2 à 3 et semble reprendre une variation en raison inverse de la température absolue.

Pour les corps paramagnétiques, dans lesquels le champ moléculaire est nul, le produit du coefficient d'aimantation spécifique $\%$ par la température absolue $\mathrm{T}$ est égal à la constante de Curie. Je vais montrer que chacune des quatre régions des fers $\alpha, \beta, \gamma, \delta$, donne une détermination de cette constante. 
Fer $\alpha$. - On peut déduire la constante de Curie, qui, par sa nature, se rapporte au fer paramagnétique, des observations sur le fer ferromagnétique. En faisant tendre $a$ et I vers zéro dans la formule (1), on a :

$$
\mathrm{I}=\mathrm{I}_{0} \cdot \frac{a}{3}=\frac{\mathrm{I}_{0} \mu \mathrm{H}}{3 r \mathrm{~T}} .
$$

ou, en multipliant haut et bas par le nombre M de molécules par centimètre cube et en remarquant que $\mathrm{M} \mu=\mathrm{I}, \mathrm{M} r \mathrm{~T}=p$, où $p$ est la pression du corps supposé gazeux :

$$
\mathrm{K}=\frac{\mathrm{I}}{\mathrm{H}}=\frac{\mathrm{I}_{0}^{2}}{3 p},
$$

ou, en appelant D la densité :

$$
\chi=\frac{\mathrm{I}_{0}^{2}}{3 p \mathrm{D}}
$$

Je vais transformer cette formule de façon à la rendre susceptible d'être étendue aux corps solides dans lesquels les molécules n'ont pas d'énergie potentielle de rotation. Appelons M le nombre des molécules par centimètre cube dans le corps considéré, $M_{0}$ le nombre des molécules dans 1 centimètre cube de gaz à la même température et sous la pression normale $p_{0}$, on aura:

$$
\chi=\frac{\mathrm{I}_{0}^{2}}{3 p_{0} \mathrm{D}} \cdot \frac{\mathrm{M}_{0}}{\mathrm{M}}
$$

En appelant $n$ le nombre d'atomes dans la molécule du corps, $a$ son poids atomique, $\delta$ la densité de l'air dans les conditions normales, il vient :

$$
\mathrm{G}=\chi_{\mathrm{T}}=\left(\frac{\mathrm{I}_{0}}{\mathrm{D}}\right)^{2} \frac{273}{3 p_{0}} \frac{n \cdot a \cdot \delta}{28,8} .
$$

Dans le cas du fer, l'intensité d'aimantation à saturation au zéro absolu $I_{0}$ se déduit de l'intensité d'aimantation à saturation à la température ordinaire au moyen de la théorie donnée ci-dessus. En admettant pour la température ordinaire le nombre de du Bois et Taylor Jones, on trouve, $\frac{I_{0}}{D}=270$, ce qui donne :

$$
\mathrm{C}=0,001646 n \text {. }
$$

Fer $\beta$. - A une température un peu supérieure à la température $\theta$ de perte du ferromagnétisme spontané, les propriétés ferroma- 
gnétiques ne se manifestent que lorsqu'un champ extérieur $\mathrm{H}_{e}$ fournit l'appoint nécessaire au champ moléculaire insuffisant. Il en résulte un coefficient d'aimantation spécifique $\gamma^{\prime}$ supérieur à celui qui se manifesterait si le champ intérieur n'existait pas. Ils sont reliés l'un à l'autre par la formule :

$$
\frac{\mathrm{T}}{\mathrm{C}}=\frac{1}{\chi}=\mathrm{D} \frac{\mathrm{H}_{e}+\mathrm{NI}}{\mathrm{I}}=\frac{\mathrm{I}}{\%}+\mathrm{DN} .
$$

Or la température $\theta$ est celle où la susceptibilité correspondant au commencement de la courbe $(f g .1)$ est égale à $\frac{1}{\mathrm{~N}}$. Il en résulte:

$$
\mathrm{CND}=\theta,
$$

et par suite :

$$
\mathrm{C}=\chi^{\prime}(\mathrm{T}-\theta),
$$

qui est, sous une autre forme, la même équation que (18).

Les expériences de Curie donnent en effet, entre $756^{\circ}$ et $820^{\circ}$, pour le coefficient d'aimantation spécifique $\chi^{\prime}$, des valeurs représentées par l'arc d'hyperbole équilatère $\mathrm{AB}$. On a:

$$
\varkappa^{\prime}=313 \times 10^{-6} \text { pour } \mathrm{T}=273^{\circ}+820^{\circ},
$$

ce qui conduit à :

$$
\mathrm{C}=0,00164 \times 2 .
$$

Il y a donc concordance complète $\dot{a}$ condition d'admettre que la molécule du fer $\beta$ se compose de deux atomes. 11 n'y a pas de raison, d'après cette théorie, pour admettre qu'elle soit différente dans le fer $\alpha$. Si cependant il y avait une modification de la molécule en un point quelconque de la région $\alpha$, il faudrait qu'elle eût lieu sans que la valeur de $I_{0}$ en fût affectée.

Fer $\gamma .-$ De $920^{\circ}$ à $1280^{\circ}$, la susceptibilité inversement proportionnelle à $\mathrm{I}$ indique l'absence de champ intérieur. Les expériences de Curie donnent à $\mathrm{T}=273^{\circ}+940^{\circ}$ :

à $\mathrm{T}=273^{\circ}+1280^{\circ}:$

$$
y=28,4 \times 10^{-6},
$$

$$
x=23,9 \times 10^{-6},
$$

d'où les valeurs assez concordantes :

$$
\mathrm{C}=0,00172 \times 2 \quad \text { et } \quad \mathrm{C}=0,00182 \times 2,
$$


c'est-ḋ-dire encore la mème constante de Curie, au degré de précision des expériences, et l'on est amené ainsi à admettre que la molécule du fer $y$ est aussi composée de deux atomes.

Fer $\delta$. - Les expériences de Curie donnent, pour $\mathrm{T}=273^{\circ}+1280^{\circ}$, $\chi=38,3 \times 10^{-6}$, d'où :

$$
\mathrm{C}=0,00198 \times 3,
$$

et pour $\mathrm{T}=273^{\circ}+1336^{\circ}, \chi=32,3 \times 10^{-6}$, d'où :

$$
\mathrm{G}=0,00173 \times 3,
$$

valeurs qui concordent encore avec les précédentes, si l'on admet que la molécule du fer ò est composée de trois atomes (1). Ces résultats me semblent pouvoir s'accorder avec ceux des intéressantes recherches de MM. Osmond et Cartaud $\left({ }^{2}\right)$ sur la cristallographie du fer. En effet, tandis que ces auteurs trouvent entre le fer $\alpha$ et $\beta$, d'une part, et le fer $\gamma$, de l'autre, une série de caractères différentiels des plus nets, les différences cristallographiques entre les états $\alpha$ et $\beta$ sont d'allure tout à fait subordonnée. Elles consistent surtout en ce que certaines particularités connues pour le fer $\alpha$ n'ont pas été observées pour le fer $\beta$; il est donc probable qu'elles n'excèdent pas celles que peut présenter le même corps à des températures aussi différentes.

Ces auteurs considèrent l'état $\alpha$ et l'état $\beta$ comme isomorphes, mais non l'état $\gamma$, et admettent que la transformation $\alpha \beta$ est progressive et réversible.

La différence de nature des deux transformations $\beta \gamma$ et yò est extrêmement intéressante. Les expériences de M. H. Le Châtelier $\left(^{(3)}\right.$ ayant montré que le passage de l'état $\beta$ à l'état $\gamma$ est accompagné d'une contraction, il semble paradoxal, à première vue, qu'à un plus grand rapprochement des aimants élémentaires puisse correspondre la disparition de leurs actions mutuelles. Mais, plus que cette particularité, l'ordre de grandeur de ces actions montre combien leur origine reste à expliquer. On a, en effet, pour le fer à la température ordi-

(1) Rappelons que, dans le langage employé ici, la molécule signifie la quantité de matière possédant l'énergie cinétique de rotation correspondant à deux degrés de liberté.

(2) Osmond et Gartaud, C. R., t. CXLII, p. 1531; 1906 ; - et Journal of the Iron and Steel Institute, $\mathrm{n}^{\circ}$ III, for 1906.

(3) Contributions à l'étude des alliages (Soc. d'Encouragement p. l'Industrie nationale, Paris, 1901, p. 411). 


$$
\begin{gathered}
\text { naire } \frac{\mathrm{I}}{\mathrm{I}_{0}}=0,91 \text { environ, d'où : } a=11,3 \text { et, par suite, } \\
\mathrm{NI}=80000000 \text { gauss. }
\end{gathered}
$$

L'énormité de ce champ est d'autant plus frappante que M. Langevin(1) a montré que le moment magnétique de l'atome d'oxygène peut fort bien n'être dû qu'à un seul corpuscule décrivant une trajectoire circulaire autour de l'atome avec une période de l'ordre de grandeur de celles des radiations du spectre visible. En reproduisant ce calcul sur l'atome de fer, on trouve une intensité d'aimantation très voisine de $I_{n}$. Mais le champ au centre de cette trajectoire est plusieurs mille fois plus faible que le champ moléculaire.

Je crois, cependant, que la théorie du champ moléculaire est appuyée par un nombre suffisant de faits pour qu'on puisse affirmer qu'elle contient une part importante de vérité et que cette difficulté d'interprétation doive être considérée moins comme une objection que comme une indication pour la recherche de nouvelles hypothèses sur la constitution de l'atome.

C'est pourquoi il peut être utile de réunir, en terminant, les renseignements que l'on possède sur le champ moléculaire.

L'hypothèse de l'uniformité de ce champ est la plus simple que l'on puisse faire, mais elle est assez invraisemblable. Sans rien changer à ce qui précède, on peut admettre que l'uniformité n'est qu'apparente et qu'elle résulte d'un effet de moyenne, soit par suite du grand nombre des molécules qui seraient contenues dans la sphère d'activité, soit, plus probablement, par suite de leur agitation thermique. Mais on peut même s'en dispenser complètement en admettant que l'élément magnétique est un aimant infiniment court, puisque le couple exercé sur un tel aimant est le même que si le champ était uniforme. On sait que dans la théorie des cycles d'aimantation donnée par Ewing, qui, le premier, a cherché à rendre compte des phénomènes résiduels par des actions mutuelles, c'est au contraire la non-uniformité du champ qui joue un rôle capital par suite de la grandeur finie des aimants élémentaires.

A propos de la transformation $\beta \gamma$, nous avons déjà remarqué que la distance des aimants élémentaires les uns des autres n'a pas l'influence prépondérante que l'on pourrait supposer. Aussi bien, relativement à cette singulière propriété du champ moléculaire, les subs-

(1) LANGEYin, loc. cit. 
tances pour lesquelles $I_{0}$ a une valeur considérable ne sont pas favorisées à l'exclusion des autres. Westman a trouvé des propriétés nettement ferromagnétiques à l'oligiste, dont l'intensité d'aimantation à saturation est voisine de 1, c'est-à-dire près de 2000 fois plus petite que celle du fer. Le cobalt, dont l'intensité d'aimantation à saturation est inférieure à celle du fer, perd son magnétisme à une température plus élevée.

On peut, pour un corps magnétique, concevoir deux manières de ne pas être ferromagnétique: ou bien le champ moléculaire n'existe pas et le corps est paramagnétique; ou bien, ce champ existant, les molécules sont bloquées par d'autres forces qui les empêchent de tourner. On peut se demander si tel ne serait pas le cas du manganèse, aux molécules duquel des métaux étrangers, l'aluminium et le cuivre, rendraient en les écartant leur mobilité.

Dans les aciers-nickel, dans les aciers au carbone, l'addition de l'élément étranger au fer abaisse la température de disparition du champ intérieur à tel point qu'il semble qu'à partir d'un certain pourcentage ils passent directement de l'état $\gamma$ à l'état $\alpha$, transformation complexe qui est irréversible. Par contre, l'addition de $20 / 0$ de silicium au fer sans carbone semble conserver l'état $\beta$ jusqu'à une température plus élevée $\left({ }^{1}\right)$. On peut espérer de l'étude quantitative des phénomènes magnétiques de ces alliages quelques éclaircissements sur la nature du champ moléculaire.

Les curieuses propriétés magnétiques des dépôts électrolytiques minces obtenus dans un champ magnétique, que M. Maurain $\left(^{2}\right)$ a découvertes, peuvent s'expliquer en admettant que, pour la première couche de fer, la sphère d'activité moléculaire étant incomplète, le champ moléculaire est moindre et, par suite, l'intensité d'aimantation plus faible que pour la matière indéfinie.

Pour le nickel, dont la première couche est plus magnétique que les suivantes, il faut admettre que les différentes parties de la sphère d'activité moléculaire contribuent au champ moléculaire par des termes de signes différents et que, pour une lame minçe, ce sont celles qui donneraient les termes négatifs qui font défaut, de sorte que le champ,

(1) Association internationale pour l'Essai des Matériaux, Congrès de Bruxelles 1906 : Osmond et Cartaud, p. $30:$ : Dans le fer presque pur de carbone, le silicium paraît supprimer presque entièrement le point $A_{3}$, c'est-à-dire le domaine du fer $\gamma$, quand il atteint une teneur de $20 / 0$ environ. "

(2) Maurain, J. de Phys., $4^{\text {e }}$ série, t. I, p. 90, 151; 1902. 
et, avec lui, l'intensité d'aimantation, est plus intense dans les premiers moments de l'électrolyse.

Ewing (1) a montré qu'une compression longitudinale diminue l'aimantation du fer et augmente celle du nickel, tandis qu'une traction longitudinale augmente l'aimantation du fer et diminue celle du nickel. Il est probable que le mécanisme de ces phénomènes, et d'autres phénomènes voisins, réside dans une altération du champ moléculaire. Si l'on remarque que la traction est accompagnée d'une contraction latérale et la compression d'une dilatation latérale, on sera tenté de rapprocher les effets inverses des déformations sur le fer et sur le nickel de leur manière d'être, également inverse, dans les expériences de M. Maurain.

On entrevoit donc, à propos des expériences de M. Maurain, la possibilité d'une généralisation de la théorie du champ moléculaire, embrassant les propriétés ferromagnétiques de la matière indéfinie et celles des couches superficielles, en relation entre elles comme la compressibilité des fluides et les phénomènes capillaires. 